between 1990 and 2008 and identifies socioeconomic factors that better explain the geographical variations of this indicator around 2008.

Methods Maternal mortality rates were calculated by level of municipal social marginalisation during the studied period. In addition, maternal mortality rates by state were calculated in 2008; by a multiple linear regression analysis, the variables that better explain interstate variations in the rate were identified.

Results The results show that in the last 20 years the maternal mortality rate has barely declined in the country (current rate is around 60 by 100000 live births), and that the ratio between the extreme strata rates-very high vs very low marginalisation -has been increased from 2.7 to 3.4 in the last 10 years. In turn, are social factors linked to poverty and social exclusion (percentage of housings with dirt floor) as well as issues related to health services (per capita public expenditure in health or percentage of population without adequate healthcare) those who better explain the variations in maternal mortality among the states. The found model explains $70 \%$ of the interstate variations of the rate.

Conclusion Previously enunciated aspects reveal the role of social conditions in maternal health and especially, in the possibility of avoiding a death from complications of pregnancy, childbirth and postpartum and demonstrate the need to change the social environment of women to reduce maternal mortality rate in Mexico.

\section{P2-514 PHYSICAL CHILD MALTREATMENT AMONG ASYLUM SEEKERS IN THE NETHERLANDS; A NATIONWIDE MEDICAL RECORDS STUDY}

doi:10.1136/jech.2011.142976m.41

\begin{abstract}
${ }^{1,2}$ S Goosen, ${ }^{*}$ A Kunst. ${ }^{1}$ Netherlands Association for Community Health Services, Utrecht, The Netherlands; ${ }^{2}$ Department of Public Health, Academic Medical Centre, University of Amsterdam, Amsterdam, The Netherlands
\end{abstract}

Introduction Refugee children in Western countries are considered to be at increased risk for child maltreatment. Data about asylum seeker children, however, are rare. The aim of our study was to assess the registered incidence of physical child maltreatment among asylum seekers in the Netherlands between 2000 and 2008, and associations with demographic and reception factors.

Methods We used the electronic database of the community health services for asylum seekers, containing demographic, reception and health data of all asylum seekers in the Netherlands between 1 January 2000 and 31 December 2008. The study includes children $<18$ years in reception with at least one parent $(N=22456)$. We used Cox regression analysis.

Results The number of physical child maltreatment cases was 182. The registered incidence was 3.7 cases per 1000 person years. Multivariate Cox regression showed an increased risk for girls $(\mathrm{RR}=1.34 ; 95 \% \mathrm{CI} 0.99$ to 1.80$)$ and children in single parent units ( $R R=1.72,95 \%$ CI 1.25 to 2.37 ). Children born in reception have a lower incidence compared to children born before arrival. Those $12-18$ years at arrival had the largest risk $(R R=2.69,95 \%$ CI 1.57 to 4.59). Incidence differences were also found between countries of origin. The highest incidence was found for children from Iran and Former Yugoslavia $(\mathrm{RR}=1.49$; $95 \% \mathrm{CI} 0.84$ to 2.65 and $\mathrm{RR}=1.30$; $95 \%$ CI 0.72 to 2.34 ). Length of stay was not an independent risk factor.

Conclusion Our study shows differences in the risk for physical child maltreatment between asylum seeker subgroups. Girls, children in single parent units and those with higher age at arrival are at increased risk. The results will contribute to priority setting in child maltreatment prevention.

\section{P2-515 INVESTIGATING VICTORIA'S INVERSE EQUITY HYPOTHESIS: THE CHANGING SOCIAL EPIDEMIOLOGY OF HIV INFECTION IN TANZANIA}

doi:10.1136/jech.2011.142976m.42

1J Hargreaves, * ${ }^{2} \mathrm{~L}$ Howe, ${ }^{1} \mathrm{E}$ Slaymaker. 'London School of Hygiene and Tropical Medicine, London, UK; ${ }^{2}$ Department of Social Medicine, University of Bristol, Bristol, UK

Introduction Cesar Victora's "inverse equity hypothesis" predicts that socioeconomic inequality in a health outcome will increase as new interventions are introduced, but suggests the gap will close over time. We explored this in relation to the HIV epidemic in Tanzania.

Methods Literature review and analysis of two nationally-representative HIV prevalence surveys conducted among adults aged 15-49 in 2003-2004 ( $N=10934)$ and 2007-2008 ( $N=15542)$. We explored whether changes over time in HIV prevalence and sexual-behaviours differed according to socioeconomic position (measured by educational attainment). In our analysis we pooled the survey data and fitted time-period*SEP interactions within appropriately adjusted regression models for each outcome.

Results Literature review suggested that when HIV prevention activities were relatively undeveloped during the 1990s, HIV prevalence was higher among more educated individuals in Tanzania. However, between 2003/4 and 2007/8 HIV prevalence was stable among those with no education (adjusted OR 2007-2008 vs 2003-2004 1.03, 0.72-1.47), whereas prevalence declined among those with secondary education (adjusted OR $0.53,0.34-0.84)$. Time-trends by education differed with respect to different aspects of sexual behaviour. There was some evidence that the association between educational attainment and recent condom use was now moving from a large poor-rich gap to a smaller one.

Conclusion The data are partially supportive of the inverse equity hypothesis. The data suggest a widening over time of the poor-rich gap in prevalent HIV infections (which may have been acquired some time ago), but there was some evidence that trends in recent condom use reflect the final "gap closing" component.

\section{P2-516 EDUCATIONAL LEVEL AND OCCUPATIONAL SOCIAL CLASS AND THE INCIDENCE OF LIFE-STYLE RELATED CHRONIC DISEASES IN THE EUROPEAN PROSPECTIVE INVESTIGATION INTO CANCER AND NUTRITION (EPIC)- POTSDAM STUDY}

doi:10.1136/jech.2011.142976m.43

${ }^{1,2} \mathrm{~A}-\mathrm{K} \mathrm{IIIner},{ }^{*}{ }^{3} \mathrm{U}$ Noethlings, ${ }^{2} \mathrm{M}$ Bergmann, ${ }^{1} \mathrm{H}$ Boeing. ${ }^{1}$ Dietary Exposure Assessment Group, International Agency for Research on Cancer, Lyon, France; ${ }^{2}$ Department of Epidemiology, German Institute of Human Nutrition, Nuthetal, Germany; ${ }^{3}$ Section for Epidemiology, Institute for Experimental Medicine, University of Kiel, Kiel, Germany

Introduction Healthy lifestyle choices, such as weight control through a balanced diet and regular physical activity, play a key role in chronic disease prevention. Underlying socioeconomic factors may mediate disease risk, but are often neglected. We aimed to investigate whether either educational level or occupational social class influences the incidence of diabetes, myocardial infarction, stroke and cancer, independently from a healthy lifestyle.

Methods We prospectively examined the associations of both indicators with incident chronic diseases in 23453 participants, aged 35-65 of the EPIC-Potsdam Study. Age- and multivariate adjusted HRs (HR, 95\% CI) were calculated. An additional systematic literature search aimed to identify how different socioeconomic indicators were related to the selected diseases. 